\title{
PULL-BACK OF QUASI-LOG STRUCTURES
}

\author{
OSAMU FUJINO
}

\begin{abstract}
We prove that the pull-back of a quasi-log scheme by a smooth quasi-projective morphism has a natural quasi-log structure. We treat an application to log Fano pairs. This paper also contains a proof of the simple connectedness of log Fano pairs with only $\log$ canonical singularities by Kento Fujita.
\end{abstract}

\section{INTRODUCTION}

The following theorem is the main result of this paper, which is natural but missing in the literature. For the precise statement, see Theorem 3.5 below.

Theorem 1.1 (Pull-back of quasi-log structures). Let $[X, \omega]$ be a quasi-log scheme and let $h: X^{\prime} \rightarrow X$ be a smooth quasi-projective morphism. Then $\left[X^{\prime}, \omega^{\prime}\right]$, where $\omega^{\prime}=h^{*} \omega \otimes \omega_{X^{\prime} / X}$ with $\omega_{X^{\prime} / X}=\operatorname{det} \Omega_{X^{\prime} / X}^{1}$, has a natural quasi-log structure induced by $h$.

In particular, if $h$ is a finite étale morphism, then $\left[X^{\prime}, \omega^{\prime}\right]$, where $\omega^{\prime}=h^{*} \omega$, has a natural quasi-log structure induced by $h$.

We make an important remark: we do not know whether Theorem 1.1 holds true or not without assuming that $h$ is quasi-projective. As an easy application of Theorem 1.1, we obtain:

Corollary 1.2. Let $[X, \omega]$ be a projective quasi-log canonical pair such that $-\omega$ is ample. Then the algebraic fundamental group of $X$ is trivial, or equivalently, $X$ has no nontrivial finite étale covers.

In view of Corollary 1.2, it is natural to conjecture:

Conjecture 1.3. Let $[X, \omega]$ be a projective quasi-log canonical pair such that $-\omega$ is ample. Then $X$ is simply connected.

In general, there exists an irreducible projective variety whose algebraic fundamental group is trivial and whose topological fundamental group is nontrivial (Example 5.4). As a special case of Conjecture 1.3, we have:

Conjecture 1.4. Let $(X, \Delta)$ be a projective semi-log canonical pair such that $-\left(K_{X}+\Delta\right)$ is ample. Then $X$ is simply connected.

It is well known that Conjecture 1.4 holds when $(X, \Delta)$ is kawamata log terminal ([T]). Kento Fujita pointed out that Conjecture 1.4 holds true when $(X, \Delta)$ is $\log$ canonical.

Theorem 1.5 (Fujita, Theorem 6.1). Let $(X, \Delta)$ be a projective log canonical pair such that $-\left(K_{X}+\Delta\right)$ is ample. Then $X$ is simply connected.

Acknowledgments. The author would like to thank Takeshi Abe, Kento Fujita, Yuichiro Hoshi, and Tetsushi Ito for answering his questions and giving him useful comments. He was partially supported by Grant-in-Aid for Young Scientists (A) 24684002, Grant-in-Aid for Scientific Research (S) 16H06337, and (B) 16H03925 from JSPS. He thanks the referees for useful comments.

Date: 2016/6/18, version 1.10 .

2010 Mathematics Subject Classification. Primary 14E30; Secondary 14J45.

Key words and phrases. quasi-log structures, log Fano pairs, fundamental groups. 
We work over $\mathbb{C}$, the complex number field, throughout this paper. We recommend the reader to see [F3] for a gentle introduction to the theory of quasi-log structures. We will never publish [F2]. Therefore, we reproduce some of the arguments from [F2] in the current paper. For the basic definitions and properties of semi-log canonial pairs, see [F6].

\section{PRELIMINARIES}

Notation 2.1. A pair $[X, \omega]$ consists of a scheme $X$ and an $\mathbb{R}$-Cartier divisor (or $\mathbb{R}$-line bundle) $\omega$ on $X$. In this paper, a scheme means a separated scheme of finite type over Spec $\mathbb{C}$. A variety is a reduced scheme.

Notation 2.2 (Divisors). Let $B_{1}$ and $B_{2}$ be two $\mathbb{R}$-Cartier divisors on a scheme $X$. Then $B_{1}$ is linearly (resp. $\mathbb{Q}$-linearly, or $\mathbb{R}$-linearly) equivalent to $B_{2}$, denoted by $B_{1} \sim B_{2}$ (resp. $B_{1} \sim_{\mathbb{Q}} B_{2}$, or $\left.B_{1} \sim_{\mathbb{R}} B_{2}\right)$ if $B_{1}=B_{2}+\sum_{i=1}^{k} r_{i}\left(f_{i}\right)$ such that $f_{i} \in \Gamma\left(X, \mathcal{K}_{X}^{*}\right)$ and $r_{i} \in \mathbb{Z}$ (resp. $r_{i} \in \mathbb{Q}$, or $r_{i} \in \mathbb{R}$ ) for every $i$. Here, $\mathcal{K}_{X}$ is the sheaf of total quotient rings of $\mathcal{O}_{X}$ and $\mathcal{K}_{X}^{*}$ is the sheaf of invertible elements in the sheaf of rings $\mathcal{K}_{X}$. We note that $\left(f_{i}\right)$ is a principal Cartier divisor associated to $f_{i}$, that is, the image of $f_{i}$ by $\Gamma\left(X, \mathcal{K}_{X}^{*}\right) \rightarrow \Gamma\left(X, \mathcal{K}_{X}^{*} / \mathcal{O}_{X}^{*}\right)$, where $\mathcal{O}_{X}^{*}$ is the sheaf of invertible elements in $\mathcal{O}_{X}$.

Let $D$ be a $\mathbb{Q}$-divisor (resp. an $\mathbb{R}$-divisor) on an equidimensional variety $X$, that is, $D$ is a finite formal $\mathbb{Q}$-linear (resp. $\mathbb{R}$-linear) combination $D=\sum_{i} d_{i} D_{i}$ of irreducible reduced subschemes $D_{i}$ of codimension one. We define the round-up $\lceil D\rceil=\sum_{i}\left\lceil d_{i}\right\rceil D_{i}$ (resp. rounddown $\lfloor D\rfloor=\sum_{i}\left\lfloor d_{i}\right\rfloor D_{i}$ ), where every real number $x,\lceil x\rceil$ (resp. $\lfloor x\rfloor$ ) is the integer defined by $x \leq\lceil x\rceil<x+1$ (resp. $x-1<\lfloor x\rfloor \leq x$ ). The fractional part $\{D\}$ of $D$ denotes $D-\lfloor D\rfloor$. We put $D^{<1}=\sum_{d_{i}<1} d_{i} D_{i}, D^{\leq 1}=\sum_{d_{i} \leq 1} d_{i} D_{i}$, and $D^{=1}=\sum_{d_{i}=1} D_{i}$. We can define $D^{\geq 1}, D^{>1}$, and so on, analogously. We call $D$ a boundary (resp. subboundary) $\mathbb{R}$-divisor if $0 \leq d_{i} \leq 1$ (resp. $d_{i} \leq 1$ ) for every $i$.

Notation 2.3 (Singularities of pairs). Let $X$ be a normal variety and let $\Delta$ be an $\mathbb{R}$ divisor on $X$ such that $K_{X}+\Delta$ is $\mathbb{R}$-Cartier. Let $f: Y \rightarrow X$ be a resolution such that $\operatorname{Exc}(f) \cup f_{*}^{-1} \Delta$, where $\operatorname{Exc}(f)$ is the exceptional locus of $f$ and $f_{*}^{-1} \Delta$ is the strict transform of $\Delta$ on $Y$, has a simple normal crossing support. We can write $K_{Y}=f^{*}\left(K_{X}+\Delta\right)+\sum_{i} a_{i} E_{i}$. We say that $(X, \Delta)$ is sub log canonical ( $s u b l c$, for short) if $a_{i} \geq-1$ for every $i$. We usually write $a_{i}=a\left(E_{i}, X, \Delta\right)$ and call it the discrepancy coefficient of $E_{i}$ with respect to $(X, \Delta)$. It is well known that there exists the largest Zariski open set $U$ of $X$ such that $\left(U,\left.\Delta\right|_{U}\right)$ is sub $\log$ canonical. If there exist a resolution $f: Y \rightarrow X$ and a divisor $E$ on $Y$ such that $a(E, X, \Delta)=-1$ and $f(E) \cap U \neq \emptyset$, then $f(E)$ is called a log canonical center (an $l c$ center, for short) with respect to $(X, \Delta)$. If $(X, \Delta)$ is sub log canonical and $\Delta$ is effective, then $(X, \Delta)$ is called log canonical (lc, for short).

We note that we can define $a\left(E_{i}, X, \Delta\right)$ in more general settings ([K2, Definition 2.4]).

Let us recall the definition of simple normal crossing pairs.

Definition 2.4 (Simple normal crossing pairs). We say that the pair $(X, D)$ is simple normal crossing at a point $a \in X$ if $X$ has a Zariski open neighborhood $U$ of $a$ that can be embedded in a smooth variety $Y$, where $Y$ has regular system of parameters $\left(x_{1}, \cdots, x_{p}, y_{1}, \cdots, y_{r}\right)$ at $a=0$ in which $U$ is defined by a monomial equation $x_{1} \cdots x_{p}=0$ and $D=\left.\sum_{i=1}^{r} \alpha_{i}\left(y_{i}=0\right)\right|_{U}$ with $\alpha_{i} \in \mathbb{R}$. We say that $(X, D)$ is a simple normal crossing pair if it is simple normal crossing at every point of $X$. We say that a simple normal crossing pair $(X, D)$ is embedded if there exists a closed embedding $\iota: X \rightarrow M$, where $M$ is a smooth variety of $\operatorname{dim} X+1$. We call $M$ the ambient space of $(X, D)$. If $(X, 0)$ is a simple normal crossing pair, then $X$ is called a simple normal crossing variety. If $X$ is a simple normal crossing variety, then $X$ has only Gorenstein singularities. Thus, it has an invertible dualizing sheaf $\omega_{X}$. Therefore, we can define the canonical divisor $K_{X}$ such that $\omega_{X} \simeq \mathcal{O}_{X}\left(K_{X}\right)$. It is a Cartier divisor on $X$ and is well-defined up to linear equivalence. 
Let $X$ be a simple normal crossing variety and let $X=\bigcup_{i \in I} X_{i}$ be the irreducible decomposition of $X$. A stratum of $X$ is an irreducible component of $X_{i_{1}} \cap \cdots \cap X_{i_{k}}$ for some $\left\{i_{1}, \cdots, i_{k}\right\} \subset I$.

Let $X$ be a simple normal crossing variety and let $D$ be a Cartier divisor on $X$. If $(X, D)$ is a simple normal crossing pair and $D$ is reduced, then $D$ is called a simple normal crossing divisor on $X$.

Let $(X, D)$ be a simple normal crossing pair. Let $\nu: X^{\nu} \rightarrow X$ be the normalization. We define $\Theta$ by the formula $K_{X^{\nu}}+\Theta=\nu^{*}\left(K_{X}+D\right)$. Then a stratum of $(X, D)$ is an irreducible component of $X$ or the $\nu$-image of a log canonical center of $\left(X^{\nu}, \Theta\right)$ (Notation 2.3). When $D=0$, this definition is compatible with the above definition of the strata of $X$. When $D$ is a boundary $\mathbb{R}$-divisor, $W$ is a stratum of $(X, D)$ if and only if $W$ is an slc stratum of $(X, D)$ ([F6, Definition 2.5]). Note that $(X, D)$ is semi-log canonical if $D$ is a boundary $\mathbb{R}$-divisor.

Notation 2.5. $\pi_{1}(X)$ denotes the topological fundamental group of $X$.

\section{Pull-BACK OF QUASI-LOG STRUCTURES}

In this section, we give a precise statement of Theorem 1.1 (Theorem 3.5). First, let us recall the definition of globally embedded simple normal crossing pairs in order to define quasi-log schemes.

Definition 3.1 (Globally embedded simple normal crossing pairs). Let $Y$ be a simple normal crossing divisor on a smooth variety $M$ and let $D$ be an $\mathbb{R}$-divisor on $M$ such that $\operatorname{Supp}(D+Y)$ is a simple normal crossing divisor on $M$ and that $D$ and $Y$ have no common irreducible components. We put $B_{Y}=\left.D\right|_{Y}$ and consider the pair $\left(Y, B_{Y}\right)$. We call $\left(Y, B_{Y}\right)$ a globally embedded simple normal crossing pair and $M$ the ambient space of $\left(Y, B_{Y}\right)$.

It is obvious that a globally embedded simple normal crossing pair is an embedded simple normal crossing pair in Definition 2.4.

Let us define quasi-log schemes. For Ambro's original definition in [A], see Definition 7.2 below.

Definition 3.2 (Quasi-log schemes). A quasi-log scheme is a scheme $X$ endowed with an $\mathbb{R}$-Cartier divisor (or $\mathbb{R}$-line bundle) $\omega$ on $X$, a proper closed subscheme $X_{-\infty} \subset X$, and a finite collection $\{C\}$ of reduced and irreducible subschemes of $X$ such that there is a proper morphism $f:\left(Y, B_{Y}\right) \rightarrow X$ from a globally embedded simple normal crossing pair satisfying the following properties:

(1) $f^{*} \omega \sim_{\mathbb{R}} K_{Y}+B_{Y}$

(2) The natural map $\mathcal{O}_{X} \rightarrow f_{*} \mathcal{O}_{Y}\left(\left\lceil-\left(B_{Y}^{<1}\right)\right\rceil\right)$ induces an isomorphism

$$
\mathcal{I}_{X_{-\infty}} \stackrel{\simeq}{\longrightarrow} f_{*} \mathcal{O}_{Y}\left(\left\lceil-\left(B_{Y}^{<1}\right)\right\rceil-\left\lfloor B_{Y}^{>1}\right\rfloor\right),
$$

where $\mathcal{I}_{X_{-\infty}}$ is the defining ideal sheaf of $X_{-\infty}$.

(3) The collection of subvarieties $\{C\}$ coincides with the image of $\left(Y, B_{Y}\right)$-strata that are not included in $X_{-\infty}$.

We simply write $[X, \omega]$ to denote the above data $\left(X, \omega, f:\left(Y, B_{Y}\right) \rightarrow X\right)$ if there is no risk of confusion. Note that a quasi-log scheme $X$ is the union of $\{C\}$ and $X_{-\infty}$. We also note that $\omega$ is called the quasi-log canonical class of $[X, \omega]$, which is defined up to $\mathbb{R}$-linear equivalence. We sometimes simply say that $[X, \omega]$ is a quasi-log pair. The subvarieties $C$ are called the qlc strata of $[X, \omega], X_{-\infty}$ is called the non-qlc locus of $[X, \omega]$, and $f:\left(Y, B_{Y}\right) \rightarrow X$ is called a quasi-log resolution of $[X, \omega]$.

Remark 3.3. Let $\operatorname{Div}(Y)$ be the group of Cartier divisors on $Y$ and let $\operatorname{Pic}(Y)$ be the Picard group of $Y$. Let $\delta_{Y}: \operatorname{Div}(Y) \otimes \mathbb{R} \rightarrow \operatorname{Pic}(Y) \otimes \mathbb{R}$ be the homomorphism induced by 
$A \mapsto \mathcal{O}_{Y}(A)$ where $A$ is a Cartier divisor on $Y$. When $\omega$ is an $\mathbb{R}$-line bundle in Definition $3.2, f^{*} \omega \sim_{\mathbb{R}} K_{Y}+B_{Y}$ means $f^{*} \omega=\delta_{Y}\left(K_{Y}+B_{Y}\right)$ in $\operatorname{Pic}(Y) \otimes \mathbb{R}$. Even when $\omega$ is an $\mathbb{R}$-line bundle, we use $-\omega$ to denote the inverse of $\omega$ in $\operatorname{Pic}(X) \otimes \mathbb{R}$ if there is no risk of confusion. If $\omega$ is an $\mathbb{R}$-Cartier divisor on $X$ in Theorem 1.1, $h^{*} \omega \otimes \operatorname{det} \Omega_{X^{\prime} / X}^{1}$ means $\delta_{X^{\prime}}\left(h^{*} \omega\right) \otimes \operatorname{det} \Omega_{X^{\prime} / X}^{1}$ in $\operatorname{Pic}\left(X^{\prime}\right) \otimes \mathbb{R}$ where $\delta_{X^{\prime}}: \operatorname{Div}\left(X^{\prime}\right) \otimes \mathbb{R} \rightarrow \operatorname{Pic}\left(X^{\prime}\right) \otimes \mathbb{R}$.

For various applications, the notion of qlc pairs is very useful.

Definition 3.4. Let $[X, \omega]$ be a quasi-log pair. We say that $[X, \omega]$ has only quasi-log canonical singularities (qlc singularities, for short) or $[X, \omega]$ is a qlc pair if $X_{-\infty}=\emptyset$.

Let us state the main theorem of this paper precisely.

Theorem 3.5 (Main theorem). Let $[X, \omega]$ be a quasi-log pair as in Definition 3.2. Let $X^{\prime}$ be a scheme and let $h: X^{\prime} \rightarrow X$ be a smooth quasi-projective morphism. Then $\left[X^{\prime}, \omega^{\prime}\right]$, where $\omega^{\prime}=h^{*} \omega \otimes \omega_{X^{\prime} / X}$ with $\omega_{X^{\prime} / X}=\operatorname{det} \Omega_{X^{\prime} / X}^{1}$, has a natural quasi-log structure induced by $h$. More precisely, we have the following:

(i) (Non-qlc locus). There is a proper closed subscheme $X_{-\infty}^{\prime} \subset X^{\prime}$.

(ii) (Quasi-log resolution). There exists a proper morphism $f^{\prime}:\left(Y^{\prime}, B_{Y^{\prime}}\right) \rightarrow X^{\prime}$ from a globally embedded simple normal crossing pair $\left(Y^{\prime}, B_{Y^{\prime}}\right)$ with $f^{\prime *} \omega^{\prime} \sim_{\mathbb{R}} K_{Y^{\prime}}+B_{Y^{\prime}}$ which defines a quasi-log structure on $\left[X^{\prime}, \omega^{\prime}\right]$ such that $\mathcal{I}_{X_{-\infty}^{\prime}}=h^{*} \mathcal{I}_{X_{-\infty}}$.

(iii) (Qlc strata). There is a finite collection $\left\{C^{\prime}\right\}$ of reduced and irreducible subschemes of $X^{\prime}$ such that $\left\{C^{\prime}\right\}=\left\{f^{-1}(C)\right\}$ and that the collection of subvarieties $\left\{C^{\prime}\right\}$ coincides with the images of $\left(Y^{\prime}, B_{Y^{\prime}}\right)$-strata that are not included in $X_{-\infty}^{\prime}$.

For the definition and basic properties of quasi-projective morphisms, see [G, Chapitre II §5.3. Morphismes quasi-projectifs].

\section{ON QUASI-LOG STRUCTURES}

Proposition 4.1 ([F2, Proposition 3.50]). Let $f: V \rightarrow W$ be a proper birational morphism between smooth varieties and let $B_{W}$ be an $\mathbb{R}$-divisor on $W$ such that $\operatorname{Supp} B_{W}$ is a simple normal crossing divisor on $W$. Assume that $K_{V}+B_{V}=f^{*}\left(K_{W}+B_{W}\right)$ and that $\operatorname{Supp} B_{V}$ is a simple normal crossing divisor on $V$. Then we have

$$
f_{*} \mathcal{O}_{V}\left(\left\lceil-\left(B_{V}^{<1}\right)\right\rceil-\left\lfloor B_{V}^{>1}\right\rfloor\right) \simeq \mathcal{O}_{W}\left(\left\lceil-\left(B_{W}^{<1}\right)\right\rceil-\left\lfloor B_{W}^{>1}\right\rfloor\right) .
$$

Furthermore, let $S$ be a simple normal crossing divisor on $W$ such that $S \subset \operatorname{Supp} B_{W}^{=1}$. Let $T$ be the union of the irreducible components of $B_{V}^{=1}$ that are mapped into $S$ by $f$. Assume that $\operatorname{Supp} f_{*}^{-1} B_{W} \cup \operatorname{Exc}(f)$ is a simple normal crossing divisor on $V$. Then we have

$$
f_{*} \mathcal{O}_{T}\left(\left\lceil-\left(B_{T}^{<1}\right)\right\rceil-\left\lfloor B_{T}^{>1}\right\rfloor\right) \simeq \mathcal{O}_{S}\left(\left\lceil-\left(B_{S}^{<1}\right)\right\rceil-\left\lfloor B_{S}^{>1}\right\rfloor\right),
$$

where $\left.\left(K_{V}+B_{V}\right)\right|_{T}=K_{T}+B_{T}$ and $\left.\left(K_{W}+B_{W}\right)\right|_{S}=K_{S}+B_{S}$.

Proof. By $K_{V}+B_{V}=f^{*}\left(K_{W}+B_{W}\right)$, we obtain

$$
K_{V}=f^{*}\left(K_{W}+B_{W}^{=1}+\left\{B_{W}\right\}\right)+f^{*}\left(\left\lfloor B_{W}^{<1}\right\rfloor+\left\lfloor B_{W}^{>1}\right\rfloor\right)-\left(\left\lfloor B_{V}^{<1}\right\rfloor+\left\lfloor B_{V}^{>1}\right\rfloor\right)-B_{V}^{=1}-\left\{B_{V}\right\} .
$$

If $a\left(\nu, W, B_{W}^{=1}+\left\{B_{W}\right\}\right)=-1$ for a prime divisor $\nu$ over $W$, then we can check that $a\left(\nu, W, B_{W}\right)=-1$ by using [KM, Lemma 2.45]. Since $f^{*}\left(\left\lfloor B_{W}^{<1}\right\rfloor+\left\lfloor B_{W}^{>1}\right\rfloor\right)-\left(\left\lfloor B_{V}^{<1}\right\rfloor+\right.$ $\left.\left\lfloor B_{V}^{>1}\right\rfloor\right)$ is Cartier, we can easily see that $f^{*}\left(\left\lfloor B_{W}^{<1}\right\rfloor+\left\lfloor B_{W}^{>1}\right\rfloor\right)=\left\lfloor B_{V}^{<1}\right\rfloor+\left\lfloor B_{V}^{>1}\right\rfloor+E$, where $E$ is an effective $f$-exceptional divisor. Thus, we obtain $f_{*} \mathcal{O}_{V}\left(\left\lceil-\left(B_{V}^{<1}\right)\right\rceil-\left\lfloor B_{V}^{>1}\right\rfloor\right) \simeq$ $\mathcal{O}_{W}\left(\left\lceil-\left(B_{W}^{<1}\right)\right\rceil-\left\lfloor B_{W}^{>1}\right\rfloor\right)$. Next, we consider the short exact sequence:

$0 \rightarrow \mathcal{O}_{V}\left(\left\lceil-\left(B_{V}^{<1}\right)\right\rceil-\left\lfloor B_{V}^{>1}\right\rfloor-T\right) \rightarrow \mathcal{O}_{V}\left(\left\lceil-\left(B_{V}^{<1}\right)\right\rceil-\left\lfloor B_{V}^{>1}\right\rfloor\right) \rightarrow \mathcal{O}_{T}\left(\left\lceil-\left(B_{T}^{<1}\right)\right\rceil-\left\lfloor B_{T}^{>1}\right\rfloor\right) \rightarrow 0$.

Since $T=f^{*} S-F$, where $F$ is an effective $f$-exceptional divisor, we can easily see that

$$
f_{*} \mathcal{O}_{V}\left(\left\lceil-\left(B_{V}^{<1}\right)\right\rceil-\left\lfloor B_{V}^{>1}\right\rfloor-T\right) \simeq \mathcal{O}_{W}\left(\left\lceil-\left(B_{W}^{<1}\right)\right\rceil-\left\lfloor B_{W}^{>1}\right\rfloor-S\right) .
$$


We note that $\left(\left\lceil-\left(B_{V}^{<1}\right)\right\rceil-\left\lfloor B_{V}^{>1}\right\rfloor-T\right)-\left(K_{V}+\left\{B_{V}\right\}+B_{V}^{=1}-T\right)=-f^{*}\left(K_{W}+B_{W}\right)$. Therefore, every associated prime of $R^{1} f_{*} \mathcal{O}_{V}\left(\left\lceil-\left(B_{V}^{<1}\right)\right\rceil-\left\lfloor B_{V}^{>1}\right\rfloor-T\right)$ is the generic point of the $f$-image of some stratum of $\left(V,\left\{B_{V}\right\}+B_{V}^{=1}-T\right)$ by [F4, Theorem 6.3 (i)].

Claim. No strata of $\left(V,\left\{B_{V}\right\}+B_{V}^{=1}-T\right)$ are mapped into $S$ by $f$.

Proof of Claim. Assume that there is a stratum $C$ of $\left(V,\left\{B_{V}\right\}+B_{V}^{=1}-T\right)$ such that $f(C) \subset$ $S$. Note that $\operatorname{Supp} f^{*} S \subset \operatorname{Supp} f_{*}^{-1} B_{W} \cup \operatorname{Exc}(f)$ and $\operatorname{Supp} B_{V}^{=1} \subset \operatorname{Supp} f_{*}^{-1} B_{W} \cup \operatorname{Exc}(f)$. Since $C$ is also a stratum of $\left(V, B_{V}^{=1}\right)$ and $C \subset \operatorname{Supp} f^{*} S$, there exists an irreducible component $G$ of $B_{V}^{=1}$ such that $C \subset G \subset \operatorname{Supp} f^{*} S$. Therefore, by the definition of $T, G$ is an irreducible component of $T$ because $f(G) \subset S$ and $G$ is an irreducible component of $B_{V}^{=1}$. So, $C$ is not a stratum of $\left(V,\left\{B_{V}\right\}+B_{V}^{=1}-T\right)$. It is a contradiction.

On the other hand, $f(T) \subset S$. Therefore, the connecting homomorphism

$$
f_{*} \mathcal{O}_{T}\left(\left\lceil-\left(B_{T}^{<1}\right)\right\rceil-\left\lfloor B_{T}^{>1}\right\rfloor\right) \rightarrow R^{1} f_{*} \mathcal{O}_{V}\left(\left\lceil-\left(B_{Z}^{<1}\right)\right\rceil-\left\lfloor B_{Z}^{>1}\right\rfloor-T\right)
$$

is a zero map by Claim. Thus, we obtain $f_{*} \mathcal{O}_{T}\left(\left\lceil-\left(B_{T}^{<1}\right)\right\rceil-\left\lfloor B_{T}^{>1}\right\rfloor\right) \simeq \mathcal{O}_{S}\left(\left\lceil-\left(B_{S}^{<1}\right)\right\rceil-\right.$ $\left.\left\lfloor B_{S}^{>1}\right\rfloor\right)$ by an easy diagram chasing. We finish the proof.

It is easy to check:

Proposition 4.2. In Proposition 4.1, let $C^{\prime}$ be a log canonical center of $\left(V, B_{V}\right)$ contained in $T$. Then $f\left(C^{\prime}\right)$ is a log canonical center of $\left(W, B_{W}\right)$ contained in $S$ or $f\left(C^{\prime}\right)$ is contained in Supp $B_{W}^{>1}$. Let $C$ be a log canonical center of $\left(W, B_{W}\right)$ contained in $S$. Then there exists a log canonical center $C^{\prime}$ of $\left(V, B_{V}\right)$ contained in $T$ such that $f\left(C^{\prime}\right)=C$.

Theorem 4.3. In Definition 3.2, we may assume that the ambient space $M$ of the globally embedded simple normal crossing pair $\left(Y, B_{Y}\right)$ is quasi-projective. In particular, $Y$ is quasiprojective.

Proof. In Definition 3.2, we may assume that $D+Y$ is an $\mathbb{R}$-divisor on a smooth variety $M$ such that $\operatorname{Supp}(D+Y)$ is a simple normal crossing divisor on $M, D$ and $Y$ have no common irreducible components, and $B_{Y}=\left.D\right|_{Y}$ as in Definition 3.1. Let $g: M^{\prime} \rightarrow M$ be a projective birational morphism from a smooth quasi-projective variety $M^{\prime}$ with the following properties:

(i) $K_{M^{\prime}}+B_{M^{\prime}}=g^{*}\left(K_{M}+D+Y\right)$,

(ii) Supp $B_{M^{\prime}}$ is a simple normal crossing divisor on $M^{\prime}$, and

(iii) Supp $g_{*}^{-1}(D+Y) \cup \operatorname{Exc}(g)$ is also a simple normal crossing divisor on $M^{\prime}$.

Let $Y^{\prime}$ be the union of the irreducible components of $B_{M^{\prime}}^{=1}$ that are mapped into $Y$ by $g$. We put $\left.\left(K_{M^{\prime}}+B_{M^{\prime}}\right)\right|_{Y^{\prime}}=K_{Y^{\prime}}+B_{Y^{\prime}}$. Then $g_{*} \mathcal{O}_{Y^{\prime}}\left(\left\lceil-\left(B_{Y^{\prime}}^{<1}\right)\right\rceil-\left\lfloor B_{Y^{\prime}}^{>1}\right\rfloor\right) \simeq \mathcal{O}_{Y}\left(\left\lceil-\left(B_{Y}^{<1}\right)\right\rceil-\right.$ $\left.\left\lfloor B_{Y}^{>1}\right\rfloor\right)$ by Proposition 4.1. This implies that $\mathcal{I}_{X_{-\infty}} \stackrel{\simeq}{\longrightarrow} f_{*} g_{*} \mathcal{O}_{Y^{\prime}}\left(\left\lceil-\left(B_{Y^{\prime}}^{<1}\right)\right\rceil-\left\lfloor B_{Y^{\prime}}^{>1}\right\rfloor\right)$. By construction, $K_{Y^{\prime}}+B_{Y^{\prime}}=g^{*}\left(K_{Y}+B_{Y}\right) \sim_{\mathbb{R}} g^{*} f^{*} \omega$. By Proposition 4.2, the collection of subvarieties $\{C\}$ in Definition 3.2 coincides with the image of $\left(Y^{\prime}, B_{Y^{\prime}}\right)$-strata that are not contained in $X_{-\infty}$. Therefore, by replacing $M$ and $\left(Y, B_{Y}\right)$ with $M^{\prime}$ and $\left(Y^{\prime}, B_{Y^{\prime}}\right)$, we may assume that the ambient space $M$ is quasi-projective.

Lemma 4.4. Let $\left(Y, B_{Y}\right)$ be a simple normal crossing pair. Let $V$ be a smooth variety such that $Y \subset V$. Then we can construct a sequence of blow-ups

$$
V_{k} \rightarrow V_{k-1} \rightarrow \cdots \rightarrow V_{0}=V
$$

with the following properties.

(1) $\sigma_{i+1}: V_{i+1} \rightarrow V_{i}$ is the blow-up along a smooth irreducible component of Supp $B_{Y_{i}}$ for every $i \geq 0$.

(2) We put $Y_{0}=Y$ and $B_{Y_{0}}=B_{Y}$. Let $Y_{i+1}$ be the strict transform of $Y_{i}$ for every $i \geq 0$. 
(3) We define $K_{Y_{i+1}}+B_{Y_{i+1}}=\sigma_{i+1}^{*}\left(K_{Y_{i}}+B_{Y_{i}}\right)$ for every $i \geq 0$.

(4) There exists an $\mathbb{R}$-divisor $D$ on $V_{k}$ such that $\left.D\right|_{Y_{k}}=B_{Y_{k}}$.

(5) $\sigma_{*} \mathcal{O}_{Y_{k}}\left(\left\lceil-\left(B_{Y_{k}}^{<1}\right)\right\rceil-\left\lfloor B_{Y_{k}}^{>1}\right\rfloor\right) \simeq \mathcal{O}_{Y}\left(\left\lceil-\left(B_{Y}^{<1}\right)\right\rceil-\left\lfloor B_{Y}^{>1}\right\rfloor\right)$, where $\sigma: V_{k} \rightarrow V_{k-1} \rightarrow$ $\cdots \rightarrow V_{0}=V$.

Proof. It is sufficient to check (5). All the other properties are obvious by the construction of the sequence of blow-ups. By an easy calculation of discrepancy coefficients similar to the proof of Proposition 4.1, we can check that

$$
\sigma_{i+1 *} \mathcal{O}_{V_{i+1}}\left(\left\lceil-\left(B_{Y_{i+1}}^{<1}\right)\right\rceil-\left\lfloor B_{Y_{i+1}}^{>1}\right\rfloor\right) \simeq \mathcal{O}_{V_{i}}\left(\left\lceil-\left(B_{Y_{i}}^{<1}\right)\right\rceil-\left\lfloor B_{Y_{i}}^{>1}\right\rfloor\right)
$$

for every $i$. This implies the desired isomorphism.

We can easily check:

Lemma 4.5. In Lemma 4.4, let $C^{\prime}$ be a stratum of $\left(Y_{k}, B_{Y_{k}}\right)$. Then $\sigma\left(C^{\prime}\right)$ is a stratum of $\left(Y, B_{Y}\right)$. Let $C$ be a stratum of $\left(Y, B_{Y}\right)$. Then there is a stratum $C^{\prime}$ of $\left(Y_{k}, B_{Y_{k}}\right)$ such that $\sigma\left(C^{\prime}\right)=C$.

The following lemma is easy but very useful (cf. [K2, Proposition 10.59]).

Lemma 4.6. Let $Y$ be a simple normal crossing variety. Let $V$ be a smooth quasi-projective variety such that $Y \subset V$. Let $\left\{P_{i}\right\}$ be any finite set of closed points of $Y$. Then we can find a quasi-projective variety $W$ such that $Y \subset W \subset V, \operatorname{dim} W=\operatorname{dim} Y+1$, and $W$ is smooth at $P_{i}$ for every $i$.

For the proof, see, for example, Step 2 in the proof of [F6, Theorem 1.2]. We note that we can not always make $W$ smooth in Lemma 4.6.

Example 4.7 ([F2, Example 3.62]). Let $V \subset \mathbb{P}^{5}$ be the Segre embedding of $\mathbb{P}^{1} \times \mathbb{P}^{2}$. In this case, there are no smooth hypersurfaces of $\mathbb{P}^{5}$ containing $V$. We can check it as follows.

If there exists a smooth hypersurface $S$ such that $V \subset S \subset \mathbb{P}^{5}$, then $\rho(V)=\rho(S)=$ $\rho\left(\mathbb{P}^{5}\right)=1$ by the Lefschetz hyperplane theorem. It is a contradiction because $\rho(V)=2$.

By the above results, we can prove the final lemma in this section.

Lemma 4.8. Let $\left(Y, B_{Y}\right)$ be a simple normal crossing pair such that $Y$ is quasi-projective. Then there exist a globally embedded simple normal crossing pair $\left(Z, B_{Z}\right)$ and a morphism $\sigma: Z \rightarrow Y$ such that $K_{Z}+B_{Z}=\sigma^{*}\left(K_{Y}+B_{Y}\right)$ and $\sigma_{*} \mathcal{O}_{Z}\left(\left\lceil-\left(B_{Z}^{<1}\right)\right\rceil-\left\lfloor B_{Z}^{>1}\right\rfloor\right) \simeq$ $\mathcal{O}_{Y}\left(\left\lceil-\left(B_{Y}^{<1}\right)\right\rceil-\left\lfloor B_{Y}^{>1}\right\rfloor\right)$. Moreover, let $C^{\prime}$ be a stratum of $\left(Z, B_{Z}\right)$. Then $\sigma\left(C^{\prime}\right)$ is a stratum of $\left(Y, B_{Y}\right)$ or $\sigma\left(C^{\prime}\right)$ is contained in $\operatorname{Supp} B_{Y}^{>1}$. Let $C$ be a stratum of $\left(Y, B_{Y}\right)$. Then there exists a stratum $C^{\prime}$ of $\left(Z, B_{Z}\right)$ such that $\sigma\left(C^{\prime}\right)=C$.

Proof. Let $V$ be a smooth quasi-projective variety such that $Y \subset V$. By Lemma 4.4 and Lemma 4.5, we may assume that there exists an $\mathbb{R}$-divisor $D$ on $V$ such that $\left.D\right|_{Y}=B_{Y}$. Then we apply Lemma 4.6. We can find a quasi-projective variety $W$ such that $Y \subset$ $W \subset V, \operatorname{dim} W=\operatorname{dim} Y+1$, and $W$ is smooth at the generic point of any stratum of $\left(Y, \operatorname{Supp} B_{Y}\right)$. Of course, we can make $W \not \subset \operatorname{Supp} D$ by the proof of Lemma 4.6. We apply Hironaka's resolution to $W$ and use Szabó's resolution lemma (see, for example, [F1, 3.5 Resolution lemma]). More precisely, we take blow-ups outside $U$, where $U$ is the largest Zariski open set of $W$ such that $\left.\left(Y, B_{Y}\right)\right|_{U}$ is a globally embedded simple normal crossing pair. Then we obtain a desired globally embedded simple normal crossing pair $\left(Z, B_{Z}\right)$. Precisely speaking, we can check that $\left(Z, B_{Z}\right)$ has the desired properties by an easy calculation of discrepancy coefficients similar to the proof of Proposition 4.1.

Theorem 4.9. In Definition 3.2, it is sufficient to assume that $\left(Y, B_{Y}\right)$ is a quasi-projective (not necessarily embedded) simple normal crossing pair. 
Proof. We only assume that $\left(Y, B_{Y}\right)$ is a simple normal crossing pair in Definition 3.2. We assume that $Y$ is quasi-projective. Then we apply Lemma 4.8 to $\left(Y, B_{Y}\right)$. Let $\sigma$ : $\left(Z, B_{Z}\right) \rightarrow\left(Y, B_{Y}\right)$ be as in Lemma 4.8. Then $\left(X, \omega, f \circ \sigma:\left(Z, B_{Z}\right) \rightarrow X\right)$ is a quasi-log scheme in the sense of Definition 3.2.

Proposition 4.10 shows that it is not so easy to apply Chow's lemma directly to make $\left(Y, B_{Y}\right)$ quasi-projective in Definition 3.2.

Proposition 4.10 ([F2, Proposition 3.65]). There exists a complete simple normal crossing variety $Y$ with the following property. If $f: Z \rightarrow Y$ is a proper surjective morphism from a simple normal crossing variety $Z$ such that $f$ is an isomorphism over the generic point of any stratum of $Y$, then $Z$ is non-projective.

Proof. We take a smooth complete non-projective toric variety $X$. We put $V=X \times \mathbb{P}^{1}$. Then $V$ is a toric variety. We consider $Y=V \backslash T$, where $T$ is the big torus of $V$. We will see that $Y$ has the desired property. By the above construction, there is an irreducible component $Y^{\prime}$ of $Y$ that is isomorphic to $X$. Let $Z^{\prime}$ be the irreducible component of $Z$ mapped onto $Y^{\prime}$ by $f$. So, it is sufficient to see that $Z^{\prime}$ is not projective. On $Y^{\prime} \simeq X$, there is a torus invariant effective one cycle $C$ such that $C$ is numerically trivial. By construction and the assumption, $g=\left.f\right|_{Z^{\prime}}: Z^{\prime} \rightarrow Y^{\prime} \simeq X$ is birational and an isomorphism over the generic point of any torus invariant curve on $Y^{\prime} \simeq X$. We note that any torus invariant curve on $Y^{\prime} \simeq X$ is a stratum of $Y$. We assume that $Z^{\prime}$ is projective, then there is a very ample effective divisor $A$ on $Z^{\prime}$ such that $A$ does not contain any irreducible components of the inverse image of $C$. Then $B=f_{*} A$ is an effective Cartier divisor on $Y^{\prime} \simeq X$ such that $\operatorname{Supp} B$ contains no irreducible components of $C$. It is a contradiction because Supp $B \cap C \neq \emptyset$ and $C$ is numerically trivial.

Proposition 4.10 is the main reason why we proved Theorem 4.3 for the proof of our main theorem: Theorem 1.1 and Theorem 3.5. Now the proof of Theorem 1.1 is almost obvious.

Proof of Theorem 3.5. Let $f:\left(Y, B_{Y}\right) \rightarrow X$ be a quasi-log resolution as in Definition 3.2. By Theorem 4.3, we may assume that $Y$ is quasi-projective. We consider the fiber product $Y^{\prime}=Y \times_{X} X^{\prime}$.

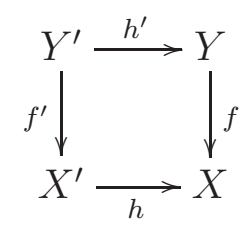

We put $B_{Y^{\prime}}=h^{\prime *} B_{Y}$. Then $\left(Y^{\prime}, B_{Y^{\prime}}\right)$ is a quasi-projective simple normal crossing pair because $h$ is a smooth quasi-projective morphism and $\left(Y, B_{Y}\right)$ is a quasi-projective simple normal crossing pair. Since $K_{Y}+B_{Y} \sim_{\mathbb{R}} f^{*} \omega$, we have

$$
f^{\prime *} \omega^{\prime}=f^{\prime} h^{*} \omega \otimes f^{\prime *} \omega_{X^{\prime} / X}=h^{\prime} f^{*} \omega \otimes \omega_{Y^{\prime} / Y} \sim_{\mathbb{R}} h^{\prime *}\left(K_{Y}+B_{Y}\right) \otimes \omega_{Y^{\prime} / Y}=K_{Y^{\prime}}+B_{Y^{\prime}} .
$$

Note that $\omega_{X^{\prime} / X}$ is trivial when $h$ is étale. By the flat base change theorem, we have

$$
\begin{aligned}
h^{*} \mathcal{I}_{X_{-\infty}} & =h^{*} f_{*} \mathcal{O}_{Y}\left(\left\lceil-\left(B_{Y}^{<1}\right)\right\rceil-\left\lfloor B_{Y}^{>1}\right\rfloor\right) \simeq f_{*}^{\prime} h^{\prime *} \mathcal{O}_{Y}\left(\left\lceil-\left(B_{Y}^{<1}\right)\right\rceil-\left\lfloor B_{Y}^{>1}\right\rfloor\right) \\
& \simeq f_{*}^{\prime} \mathcal{O}_{Y^{\prime}}\left(\left\lceil-\left(B_{Y^{\prime}}^{<1}\right)\right\rceil-\left\lfloor B_{Y^{\prime}}^{>1}\right\rfloor\right) .
\end{aligned}
$$

Finally, by Theorem 4.9, we may assume that $\left(Y^{\prime}, B_{Y^{\prime}}\right)$ is a globally embedded simple normal crossing pair. Therefore, $\left(X^{\prime}, \omega^{\prime}, f^{\prime}:\left(Y^{\prime}, B_{Y^{\prime}}\right) \rightarrow X^{\prime}\right)$ gives us the desired quasi$\log$ structure. 


\section{An application to quasi-log CANONicAl FANO VARIETiES}

Let us recall the vanishing theorem for projective qlc pairs.

Theorem 5.1 (Vanishing theorem for qlc pairs). Let $[X, \omega]$ be a projective qlc pair and let $L$ be a Cartier divisor on $X$ such that $L-\omega$ is ample. Then $H^{i}\left(X, \mathcal{O}_{X}(L)\right)=0$ for every $i>0$.

We give a proof of Theorem 5.1 for the reader's convenience.

Proof. Let $f:\left(Y, B_{Y}\right) \rightarrow X$ be a quasi-log resolution as in Definition 3.2. Since $[X, \omega]$ is qle, $B_{Y}=B_{Y}^{\leq 1}$ holds. Then

$$
f^{*}(L-\omega) \sim_{\mathbb{R}} f^{*} L-\left(K_{Y}+B_{Y}\right)=f^{*} L+\left\lceil-\left(B_{Y}^{<1}\right)\right\rceil-\left(K_{Y}+\left\{B_{Y}\right\}+B_{Y}^{=1}\right)
$$

because $B_{Y}=B_{\bar{Y}}^{\leq 1}$. Therefore, we have $H^{i}\left(X, f_{*} \mathcal{O}_{Y}\left(f^{*} L+\left\lceil-\left(B_{Y}^{<1}\right)\right\rceil\right)\right)=0$ for every $i>0$ by $\left[\right.$ F5, Theorem 1.1 (ii)]. Note that $f_{*} \mathcal{O}_{Y}\left(f^{*} L+\left\lceil-\left(B_{Y}^{<1}\right)\right\rceil\right) \simeq \mathcal{O}_{X}(L) \otimes f_{*} \mathcal{O}_{Y}\left(\left\lceil-\left(B_{Y}^{<1}\right)\right\rceil\right) \simeq$ $\mathcal{O}_{X}(L)$ because $X_{-\infty}=\emptyset$. This implies that $H^{i}\left(X, \mathcal{O}_{X}(L)\right)=0$ for every $i>0$.

By combining Theorem 5.1 with Theorem 3.5, we can easily check Corollary 1.2.

Proof of Corollary 1.2. Without loss of generality, we may assume that $X$ is connected. Since $-\omega$ is ample, $H^{i}\left(X, \mathcal{O}_{X}\right)=0$ for every $i>0$ by Theorem 5.1. Therefore, we have $\chi\left(X, \mathcal{O}_{X}\right)=1$. Assume there exists a nontrivial finite étale morphism $f: \tilde{X} \rightarrow X$ from a connected scheme $\widetilde{X}$. By Theorem 3.5 , the pair $[\widetilde{X}, \widetilde{\omega}]$, where $\widetilde{\omega}=f^{*} \omega$, is a qlc pair such that $-\widetilde{\omega}$ is ample. Thus, $H^{i}\left(\widetilde{X}, \mathcal{O}_{\tilde{X}}\right)=0$ for every $i>0$ by Theorem 5.1 again. This implies $\chi\left(\widetilde{X}, \mathcal{O}_{\widetilde{X}}\right)=1$. By the Riemann-Roch formula ([Ft, Example 18.3.9]), we have $\chi\left(\widetilde{X}, \mathcal{O}_{\tilde{X}}\right)=\operatorname{deg} f \cdot \chi\left(X, \mathcal{O}_{X}\right)$. Therefore, we obtain $\operatorname{deg} f=1$, a contradiction. This means that $X$ has no nontrivial finite étale covers, or equivalently, the algebraic fundamental group of $X$ is trivial.

As a direct consequence of Corollary 1.2 and the main theorem of [F6], we have:

Corollary 5.2. Let $(X, \Delta)$ be a projective semi-log canonical pair such that $-\left(K_{X}+\Delta\right)$ is ample. Then the algebraic fundamental group of $X$ is trivial.

Proof. By [F6], $\left[X, K_{X}+\Delta\right]$ has a natural quasi-log structure with only qlc singularities. Therefore, Corollary 5.2 is a special case of Corollary 1.2.

Note that a union of some slc strata of a log Fano pair with only semi-log canonical singularities is a quasi-log canonical Fano variety by Example 5.3.

Example 5.3. Let $(X, \Delta)$ be a connected projective semi-log canonical pair such that $-\left(K_{X}+\Delta\right)$ is ample. Let $W$ be a union of some slc strata of $(X, \Delta)$ with the reduced scheme structure. Then $[W, \omega]$, where $\omega=\left.\left(K_{X}+\Delta\right)\right|_{W}$, is a projective qlc pair such that $-\omega$ is ample by adjunction $([\mathrm{F} 6$, Theorem 1.13]). By [F6, Theorem 1.11], we obtain $H^{1}\left(X, \mathcal{I}_{W}\right)=0$ where $\mathcal{I}_{W}$ is the defining ideal sheaf of $W$ on $X$. Therefore, we obtain $H^{0}\left(W, \mathcal{O}_{W}\right)=\mathbb{C}$ by the surjection $\mathbb{C}=H^{0}\left(X, \mathcal{O}_{X}\right) \rightarrow H^{0}\left(W, \mathcal{O}_{W}\right)$. This implies that $W$ is connected.

The author learned the following example from Tetsushi Ito.

Example 5.4 (Topological versus algebraic). We consider the Higman group $G$. It is generated by 4 elements $a, b, c, d$ with the relations

$$
a^{-1} b a=b^{2}, \quad b^{-1} c b=c^{2}, \quad c^{-1} d c=d^{2}, \quad d^{-1} a d=a^{2} .
$$

It is well known that $G$ has no nontrivial finite quotients. By [S, Theorem 12.1], there is an irreducible projective variety $X$ such that $\pi_{1}(X) \simeq G$. In this case, the algebraic fundamental group of $X$, which is the profinite completion of $\pi_{1}(X)$, is trivial. 
Example 5.4 shows that Conjecture 1.3 does not directly follow from Corollary 1.2. We give a nontrivial example of reducible log Fano pairs with only semi-log canonical singularities.

Example 5.5. We consider the lattice $N=\mathbb{Z}^{3}$. Let $n$ be an integer with $n \geq 3$. We consider a convex polyhedron $P$ in $N_{\mathbb{R}}=N \otimes \mathbb{R} \simeq \mathbb{R}^{3}$ whose vertices are $v_{0}, v_{1}, \ldots, v_{n} \in N$ such that $v_{0}=(0,0,-1)$ and that the third coordinates of $v_{1}, \ldots, v_{n}$ are 1 . Assume that $P$ contains $(0,0,0)$ in its interior. Then the cones spanned by $(0,0,0)$ and faces of $P$ subdivide $\mathbb{R}^{3}$ into $n+1$ three-dimensional cones. This subdivision of $\mathbb{R}^{3}$ corresponds to a complete toric threefold $X$. Then we have the following properties.

(1) $-K_{X}$ is ample since $P$ is convex.

(2) $D_{0} \sim D_{1}+\cdots+D_{n}$ and $D_{0}$ is $\mathbb{Q}$-Cartier, where $D_{i}$ is the torus invariant prime divisor on $X$ associated to $v_{i}$ for every $i$.

(3) Let $x \in X$ be the torus invariant closed point associated to the cone spanned by $v_{1}, v_{2}, \ldots, v_{n}$. Then $X \backslash x$ is $\mathbb{Q}$-factorial, but $X$ is not $\mathbb{Q}$-factorial when $n \geq 4$.

(4) We put $\Delta=D_{1}+\cdots+D_{n}$. Then $(X, \Delta)$ is a log canonical Fano threefold. Note that $-\left(K_{X}+\Delta\right) \sim D_{0}$.

(5) We put $W=\lfloor\Delta\rfloor=\Delta$ and $K_{W}+\Delta_{W}=\left.\left(K_{X}+\Delta\right)\right|_{W}$. Then $\left(W, \Delta_{W}\right)$ is a twodimensional log Fano pair with only semi-log canonical singularities. Note that $W$ is Cohen-Macaulay since $W$ is $\mathbb{Q}$-Cartier.

This $W$ shows that the number of irreducible components of log Fano pairs with only semi-log canonical singularities is not bounded.

We recommend the reader who can read Japanese to see [F7] for some related topics and open problems on singular Fano varieties.

\section{Simple Connectedness of LOG CANONicAl LOG FAnO PAIRS}

In this section, we prove that a log Fano pair with only log canonical singularities is always simply connected. Theorem 6.1 is Fujita's answer to the author's question.

Theorem 6.1 (Fujita). Let $(X, \Delta)$ be a projective log canonical pair such that $-\left(K_{X}+\Delta\right)$ is ample. Then $X$ is simply connected.

Proof. First of all, we may assume that $X$ is connected. Without loss of generality, we may assume that $\Delta$ is a $\mathbb{Q}$-divisor by perturbing $\Delta$ slightly. Then, by [HM, Corollary 1.3 (2)], $X$ is rationally chain connected. Since $X$ is normal and rationally chain connected, $\pi_{1}(X)$ is finite $([\mathrm{K} 1,4.13$ Theorem $])$. Let $f: \tilde{X} \rightarrow X$ be the universal cover of $X$. Since $\pi_{1}(X)$ is finite, $f$ is finite and étale. It is obvious that $(\widetilde{X}, \widetilde{\Delta})$ is log canonical and $-\left(K_{\widetilde{X}}+\widetilde{\Delta}\right)$ is ample, where $K_{\widetilde{X}}+\widetilde{\Delta}=f^{*}\left(K_{X}+\Delta\right)$. By [F4, Theorem 8.1], we have $H^{i}\left(X, \mathcal{O}_{X}\right)=H^{i}\left(\widetilde{X}, \mathcal{O}_{\tilde{X}}\right)=0$ for every $i>0$. This implies $\chi\left(X, \mathcal{O}_{X}\right)=\chi\left(\widetilde{X}, \mathcal{O}_{\tilde{X}}\right)=1$. On the other hand, $\chi\left(\widetilde{X}, \mathcal{O}_{\widetilde{X}}\right)=\operatorname{deg} f \cdot \chi\left(X, \mathcal{O}_{X}\right)$ holds by the Riemann-Roch formula ([Ft, Example 18.3.9]). Thus we obtain $\operatorname{deg} f=1$. Therefore, $X$ is simply connected.

Remark 6.2. By [HM, Corollary 1.3 (2)], we can easily see that a log Fano pair with only semi-log canonical singularities is rationally chain connected. However, [K1, 4.13 Theorem] does not always hold for non-normal rationally chain connected varieties. Note that a nodal rational curve $C$ is rationally chain connected such that $\pi_{1}(C)$ is infinite. Therefore, the proof of Theorem 6.1 does not work for log Fano pairs with only semi-log canonical singularities.

The following well-known example shows some subtleties on log Fano pairs with only log canonical singularities. Example 6.3 says that Theorem 6.1 does not always hold when $-\left(K_{X}+\Delta\right)$ is only nef and big. 
Example 6.3. Let $C \subset \mathbb{P}^{2}$ be a smooth cubic curve and let $X \subset \mathbb{P}^{3}$ be the cone over $C \subset \mathbb{P}^{2}$. Then $X$ is a Gorenstein log canonical surface such that $-K_{X}$ is ample. It is easy to see that $X$ is rationally chain connected and that $\pi_{1}(X)=\{1\}$ by Theorem 6.1 . Let $f: Y \rightarrow X$ be the blow-up at $P$ where $P$ is the vertex of $X$. Then $K_{Y}+E=f^{*} K_{X}$. The pair $(Y, E)$ is purely $\log$ terminal and $-\left(K_{Y}+E\right)$ is big and semiample. Note that the exceptional curve $E$ is isomorphic to $C$ and that $Y$ is a $\mathbb{P}^{1}$-bundle over $C$. Therefore, it is easy to see that $Y$ is not rationally chain connected and $\pi_{1}(Y) \neq\{1\}$.

Example 6.4 is a nontrivial example of irreducible non-normal semi-log canonical Fano varieties.

Example 6.4. We put $X=\left(x^{2} w-z y^{2}=0\right) \subset \mathbb{P}^{3}$. Then $X$ is a Gorenstein Fano variety with only semi-log canonical singularities. Note that $X$ is irreducible and non-normal. By using the van Kampen theorem, we see that $\pi_{1}(X)=\{1\}$.

\section{Appendix: Ambro's original definition}

In this section, we prove that our definition of quasi-log schemes (Definition 3.2) is equivalent to Ambro's original definition in $[\mathrm{A}]$.

First, let us recall the definition of normal crossing pairs. We need it for Ambro's original definition of quasi-log schemes in $[\mathrm{A}]$.

Definition 7.1 (Normal crossing pairs). A variety $X$ has normal crossing singularities if, for every closed point $x \in X$,

$$
\widehat{\mathcal{O}}_{X, x} \simeq \frac{\mathbb{C}\left[\left[x_{0}, \cdots, x_{N}\right]\right]}{\left(x_{0} \cdots x_{k}\right)}
$$

for some $0 \leq k \leq N$, where $N=\operatorname{dim} X$. Let $X$ be a normal crossing variety. We say that a reduced divisor $D$ on $X$ is normal crossing if, in the above notation, we have

$$
\widehat{\mathcal{O}}_{D, x} \simeq \frac{\mathbb{C}\left[\left[x_{0}, \cdots, x_{N}\right]\right]}{\left(x_{0} \cdots x_{k}, x_{i_{1}} \cdots x_{i_{l}}\right)}
$$

for some $\left\{i_{1}, \cdots, i_{l}\right\} \subset\{k+1, \cdots, N\}$. A stratum of $X$ is an irreducible component of $X$ or the $\nu$-image of a $\log$ canonical center of $\left(X^{\nu}, \Xi\right)$, where $\nu: X^{\nu} \rightarrow X$ is the normalization and $K_{X^{\nu}}+\Xi=\nu^{*} K_{X}$. A permissible Cartier divisor on $X$ is a Cartier divisor on $X$ whose support contains no strata of $X$. A permissible $\mathbb{R}$-Cartier divisor is a finite $\mathbb{R}$-linear combination of permissible Cartier divisors on $X$. We say that the pair $(X, B)$ is a normal crossing pair if the following conditions are satisfied.

(1) $X$ is a normal crossing variety, and

(2) $B$ is a permissible $\mathbb{R}$-Cartier divisor whose support is normal crossing on $X$.

We say that a normal crossing pair $(X, B)$ is embedded if there exists a closed embedding $\iota: X \rightarrow M$, where $M$ is a smooth variety of dimension $\operatorname{dim} X+1$. We call $M$ the ambient space of $(X, B)$. We put

$$
K_{X^{\nu}}+\Theta=\nu^{*}\left(K_{X}+B\right),
$$

where $\nu: X^{\nu} \rightarrow X$ is the normalization of $X$. A stratum of $(X, B)$ is an irreducible component of $X$ or the $\nu$-image of some log canonical center of $\left(X^{\nu}, \Theta\right)$ on $X$.

It is obvious that a simple normal crossing pair in Definition 2.4 is a normal crossing pair in Definition 7.1. Note that the differences between normal crossing varieties and simple normal crossing varieties sometimes cause some subtle troubles. For the details, see, for example, [F1, 3.6 Whitney umbrella].

Let us recall Ambro's original definition of quasi-log schemes in [A]. 
Definition 7.2 (Quasi-log schemes). A quasi-log scheme is a scheme $X$ endowed with an $\mathbb{R}$-Cartier divisor (or $\mathbb{R}$-line bundle) $\omega$, a proper closed subscheme $X_{-\infty} \subset X$, and a finite collection $\{C\}$ of reduced and irreducible subschemes of $X$ such that there is a proper morphism $f:\left(Y, B_{Y}\right) \rightarrow X$ from an embedded normal crossing pair satisfying the following properties:

(1) $f^{*} \omega \sim_{\mathbb{R}} K_{Y}+B_{Y}$.

(2) The natural map $\mathcal{O}_{X} \rightarrow f_{*} \mathcal{O}_{Y}\left(\left\lceil-\left(B_{Y}^{<1}\right)\right\rceil\right)$ induces an isomorphism

$$
\mathcal{I}_{X_{-\infty}} \stackrel{\simeq}{\longrightarrow} f_{*} \mathcal{O}_{Y}\left(\left\lceil-\left(B_{Y}^{<1}\right)\right\rceil-\left\lfloor B_{Y}^{>1}\right\rfloor\right),
$$

where $\mathcal{I}_{X_{-\infty}}$ is the defining ideal sheaf of $X_{-\infty}$.

(3) The collection of subvarieties $\{C\}$ coincides with the image of $\left(Y, B_{Y}\right)$-strata that are not included in $X_{-\infty}$.

In Definition 3.2, we assume that $\left(Y, B_{Y}\right)$ is a globally embedded simple normal crossing pair. On the other hand, in Definition 7.2 , we only assume that $\left(Y, B_{Y}\right)$ is an embedded normal crossing pair.

Remark 7.3 (Schemes versus varieties). A quasi-log scheme is called a quasi-log variety in $[\mathrm{A}]$. However, $X$ is not always reduced when $X_{-\infty} \neq \emptyset$. Note that $X$ is reduced when $X_{-\infty}=\emptyset$.

Example 7.4 ([A, Examples 4.3.4]). Let $X$ be an effective Cartier divisor on a smooth variety $M$ such that Supp $X$ is a simple normal crossing divisor. Assume that $Y$, the reduced part of $X$, is non-empty. We put $\omega=\left.\left(K_{M}+X\right)\right|_{X}$. Let $X_{-\infty}$ be the union of the non-reduced components of $X$. We put $K_{Y}+B_{Y}=\left.\left(K_{M}+X\right)\right|_{Y}$. Let $f: Y \rightarrow X$ be the closed embedding. Then $\left(X, \omega, f:\left(Y, B_{Y}\right) \rightarrow X\right)$ is a quasi-log scheme. Note that $X$ has non-reduced irreducible components if $X_{-\infty} \neq \emptyset$. We also note that $f$ is not surjective if $X_{-\infty} \neq \emptyset$.

Lemma 7.5 is essentially the same as Ambro's embedded log transformations in [A].

Lemma 7.5. Let $\left(Y, B_{Y}\right)$ be an embedded normal crossing pair and let $M$ be the ambient space of $\left(Y, B_{Y}\right)$. Then there are a projective surjective morphism $\sigma: M^{\prime} \rightarrow M$ from a smooth variety $M^{\prime}$ such that $\sigma$ is a composition of blow-ups and a simple normal crossing pair $\left(Z, B_{Z}\right)$ embedded into $M^{\prime}$ with the following properties.

(i) $\sigma: Z \rightarrow Y$ is surjective and $K_{Z}+B_{Z}=\sigma^{*}\left(K_{Y}+B_{Y}\right)$.

(ii) $\sigma_{*} \mathcal{O}_{Z}\left(\left\lceil-\left(B_{Z}^{<1}\right)\right\rceil-\left\lfloor B_{Z}^{>1}\right\rfloor\right) \simeq \mathcal{O}_{Y}\left(\left\lceil-\left(B_{Y}^{<1}\right)\right\rceil-\left\lfloor B_{Y}^{>1}\right\rfloor\right)$.

(iii) Let $C^{\prime}$ be a stratum of $\left(Z, B_{Z}\right)$. Then $\sigma\left(C^{\prime}\right)$ is a stratum of $\left(Y, B_{Y}\right)$ or is contained in Supp $B_{Y}^{>1}$. Let $C$ be a stratum of $\left(Y, B_{Y}\right)$. Then there is a stratum $C^{\prime}$ of $\left(Z, B_{Z}\right)$ such that $\sigma\left(C^{\prime}\right)=C$.

Proof. First, we can construct a sequence of blow-ups $M_{k} \rightarrow M_{k-1} \rightarrow \cdots \rightarrow M_{0}=M$ with the following properties.

(a) $\sigma_{i+1}: M_{i+1} \rightarrow M_{i}$ is the blow-up along a smooth stratum of $Y_{i}$ for every $i$.

(b) We put $Y_{0}=Y, B_{Y_{0}}=B_{Y}$, and $Y_{i+1}=\sigma_{i+1}^{-1}\left(Y_{i}\right)$ with the reduced scheme structure.

(c) $Y_{k}$ is a simple normal crossing divisor on $M_{k}$.

We can check that $K_{Y_{i+1}}=\sigma_{i+1}^{*} K_{Y_{i}}$ for every $i$ by construction. We can directly check that $R^{1} \sigma_{i+1 *} \mathcal{O}_{M_{i+1}}\left(-Y_{i+1}\right)=0$ and $\sigma_{i+1 *} \mathcal{O}_{M_{i+1}}\left(-Y_{i+1}\right) \simeq \mathcal{O}_{M_{i}}\left(-Y_{i}\right)$ for every $i$. Therefore, by the diagram:

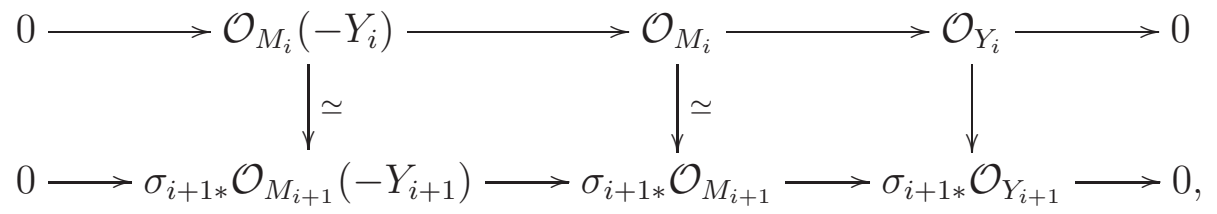


we obtain $\sigma_{i+1 *} \mathcal{O}_{Y_{i+1}} \simeq \mathcal{O}_{Y_{i}}$ for every $i$. We put $B_{Y_{i+1}}=\sigma_{i+1}^{*} B_{Y_{i}}$ for every $i$. Then, by replacing $\left(Y, B_{Y}\right)$ and $M$ with $\left(Y_{k}, B_{Y_{k}}\right)$ and $M_{k}$, we may assume that $Y$ is a simple normal crossing divisor on $M$.

Next, we can construct a sequence of blow-ups $M_{k} \rightarrow M_{k-1} \rightarrow \cdots \rightarrow M_{0}=M$ with the following properties.

(1) $\sigma_{i+1}: M_{i+1} \rightarrow M_{i}$ is the blow-up along a smooth stratum of $\left(Y_{i}\right.$, Supp $\left.B_{Y_{i}}\right)$ contained in Supp $B_{Y_{i}}$ for every $i$.

(2) We put $Y_{0}=Y$ and $B_{Y_{0}}=B_{Y}$. Let $Y_{i+1}$ be the strict transform of $Y_{i}$ on $M_{i+1}$ for every $i$.

(3) We put $K_{Y_{i+1}}+B_{Y_{i+1}}=\sigma_{i+1}^{*}\left(K_{Y_{i}}+B_{Y_{i}}\right)$ for every $i$.

(4) Supp $B_{Y_{k}}$ is a simple normal crossing divisor on $Y_{k}$.

Finally, by construction, we can check the properties (i), (ii), and (iii) for $\sigma: M_{k} \rightarrow M$ and $\left(Y_{k}, B_{Y_{k}}\right)$ by an easy calculation of discrepancy coefficients similar to the proof of Proposition 4.1.

Proposition 7.6. Assume that $\left(Y, B_{Y}\right)$ is an embedded simple normal crossing pair in Definition 7.2. Let $M$ be the ambient space of $\left(Y, B_{Y}\right)$. Then, by taking some sequence of blow-ups of $M$, we may further assume that $\left(Y, B_{Y}\right)$ is a globally embedded simple normal crossing pair in Definition \%.2.

Proof. It is sufficient to apply Lemma 4.4 and Lemma 4.5 by putting $V=M$. If $B_{Y}=B_{Y}^{\leq 1}$, then this proposition is nothing but [F6, Lemma 3.3].

Therefore, by Lemma 7.5 and Proposition 7.6, Definition 3.2 is equivalent to Ambro's original definition of quasi-log schemes: Definition 7.2.

Theorem 7.7. Definition 3.2 is equivalent to Definition \%.2.

\section{REFERENCES}

[A] F. Ambro, Quasi-log varieties, Tr. Mat. Inst. Steklova 240 (2003), Biratsion. Geom. Linein. Sist. Konechno Porozhdennye Algebry, 220-239; translation in Proc. Steklov Inst. Math. 2003, no. 1 (240), 214-233.

[F1] O. Fujino, What is log terminal?, Flips for 3-folds and 4-folds, 49-62, Oxford Lecture Ser. Math. Appl., 35, Oxford Univ. Press, Oxford, 2007.

[F2] O. Fujino, Introduction to the log minimal model program for log canonical pairs, preprint (2008).

[F3] O. Fujino, Introduction to the theory of quasi-log varieties, Classification of algebraic varieties, 289-303, EMS Ser. Congr. Rep., Eur. Math. Soc., Zürich, 2011.

[F4] O. Fujino, Fundamental theorems for the log minimal model program, Publ. Res. Inst. Math. Sci. 47 (2011), no. 3, 727-789.

[F5] O. Fujino, Vanishing theorems, to appear in Adv. Stud. Pure Math.

[F6] O. Fujino, Fundamental theorems for semi log canonical pairs, Algebr. Geom. 1 (2014), no. 2, 194-228.

[F7] O. Fujino, Some problems on Fano varieties (Japanese), Sūrikaisekikenkyūsho Kōkyūroku No. 1897 (2014), 43-70.

[Ft] W. Fulton, Intersection theory, Second edition. Results in Mathematics and Related Areas. 3rd Series. A Series of Modern Surveys in Mathematics, 2. Springer-Verlag, Berlin, 1998.

[G] A. Grothendieck, Éléments de géométrie algébrique. II. Étude globale élémentaire de quelques classes de morphismes, Inst. Hautes Études Sci. Publ. Math. No. 8 (1961).

[HM] C. D. Hacon, J. McKernan, On Shokurov's rational connectedness conjecture, Duke Math. J. 138 (2007), no. 1, 119-136.

[K1] J. Kollár, Shafarevich maps and automorphic forms, M. B. Porter Lectures. Princeton University Press, Princeton, NJ, 1995.

[K2] J. Kollár, Singularities of the minimal model program, With a collaboration of Sándor Kovács. Cambridge Tracts in Mathematics, 200. Cambridge University Press, Cambridge, 2013.

[KM] J. Kollár, S. Mori, Birational geometry of algebraic varieties, With the collaboration of C. H. Clemens and A. Corti. Translated from the 1998 Japanese original. Cambridge Tracts in Mathematics, 134. Cambridge University Press, Cambridge, 1998. 
[S] C. Simpson, Local systems on proper algebraic $V$-manifolds, Pure Appl. Math. Q. 7 (2011), no. 4, Special Issue: In memory of Eckart Viehweg, 1675-1759.

[T] S. Takayama, Simple connectedness of weak Fano varieties, J. Algebraic Geom. 9 (2000), no. 2, 403-407.

Department of Mathematics, Graduate School of Science, Osaka University, ToyonAKA, OSAKA 560-0043, JAPAN

E-mail address: fujino@math.sci.osaka-u.ac.jp 